\title{
Fatal perinatal nephropathy with onset in intrauterine life
}

\author{
R J Scott, M Rochefort
}

\begin{abstract}
A girl, born at 29 weeks' gestation, died of renal failure aged 16 days. Postmortem histology showed diffuse mesangial sclerosis with failure of development of the cortex. This is an unusual cause of neonatal renal failure, and it demonstrates the effect of disease arising in utero and influencing the development of the kidney.
\end{abstract}

(Arch Dis Child 1992;67:1212-3)

Renal failure in neonates is rarely due to primary renal disease. We present a case of a neonate, born at 29 weeks' gestation, in whom renal failure led to death within 16 days. The pathological findings demonstrate an effect of the disease process on the maturation of the kidney.

\section{Case report}

The mother was a 28 year old white primigravida. She was negative for syphilis antibodies and had no history of renal or autoimmune disease or of hypertension before pregnancy. The parents were unrelated; the father had sarcoidosis. Serum $\alpha$-fetoprotein at week 18 was raised at $3.2 \times$ median for the gestation. Fetal ultrasound was normal.

Severe hypertension developed during the last four days before delivery, and an emergency caesarean section was performed at 29 weeks after an antepartum haemorrhage.

The infant girl weighed $1160 \mathrm{~g}$ and required resuscitation at birth. Her Apgar scores were 2 at 1 minute and 4 at 5 minutes; she was also ventilated.

Respiratory distress was present for the first three days and was complicated by a pneumothorax. She was treated with pancuronium, surfactant, piperacillin, and gentamicin. Her blood pressure was well maintained. Although the infant was mildly oedematous with an albumin concentration of $27 \mathrm{~g} / 1$, she passed urine at $5 \mathrm{ml} / \mathrm{kg} /$ hour for the first 72 hours. Urinalysis was negative and the specific gravity was below 1012. An umbilical catheter was removed at 24 hours because of left leg mottling.

At 96 hours proteinuria and haematuria were detected, but proteinuria was transient and not quantified. Her urea concentration rose to $9 \mathrm{mmol} / 1$ from $4.8 \mathrm{mmol} / 1$ at birth; creatinine was static from birth at $109 \mathrm{mmol} / \mathrm{l}$ until day 7 when it increased progressively. At that time the infant became oliguric and hypertensive and gained weight rapidly. Cardiac and renal ultrasound were normal. The baby rapidly became anuric, and peritoneal dialysis was commenced. The baby died at 16 days of respiratory failure.

Chromosome analysis was not performed and specific genetic counselling was not undertaken. A normal female sibling was born in January 1992.

\section{POSTMORTEM EXAMINATION}

A limited examination was carried out and the infant was found to be normally formed, weight $1240 \mathrm{~g}$, on the 10th centile; she had normal genitalia. Internal examination showed peritoneal oedema, bile stained ascites, a congested liver, small adrenals, and two small gastric ulcers. The kidneys were enlarged, pale, and oedematous and their combined weight was $21.3 \mathrm{~g}$ (normal combined weight $=12.9$ $( \pm 33.9) \mathrm{g})$ for a $1250 \mathrm{~g}$ fetus. ${ }^{1}$ There were no cysts. The ureters, bladder, and urethra were normal. The bladder was empty.

The placenta (weight $215 \mathrm{~g}$ ) was not oedematous and showed no evidence of abruption.

MICROSCOPY OF KIDNEY (FIG 1 AND 2A)

Despite the large size of the kidneys there were only 4-5 layers of glomeruli. The nephrogenic blastema was disordered and reduced in thickness. The superficial glomeruli were small and immature, with prominent primitive epithelial cells. The deepest glomeruli had developed appropriately, but they were enlarged and showed mesangial sclerosis. There was no increase in cellularity and no crescent formation. The tubules were dilated by a mass of amorphous material that stained positive with periodic acid Schiff; they were not dysplastic and there was no evidence of acute tubular necrosis. This tubular dilation appears to be the cause of the increased renal weight.

Immunostaining showed peripheral $\operatorname{IgM}$ and C3 involving all the glomeruli, including immature glomeruli that could barely have acquired function.

\section{ELECTRON MICROSCOPY (FIG 2B)}

In the larger glomeruli the epithelial cells were separated from the basement membrane, with some floating free in Bowman's space. Beneath these cells was a mass of amorphous material, which was not electron dense, and therefore did not represent an immune complex deposit. The basement membrane was tortuous, with 


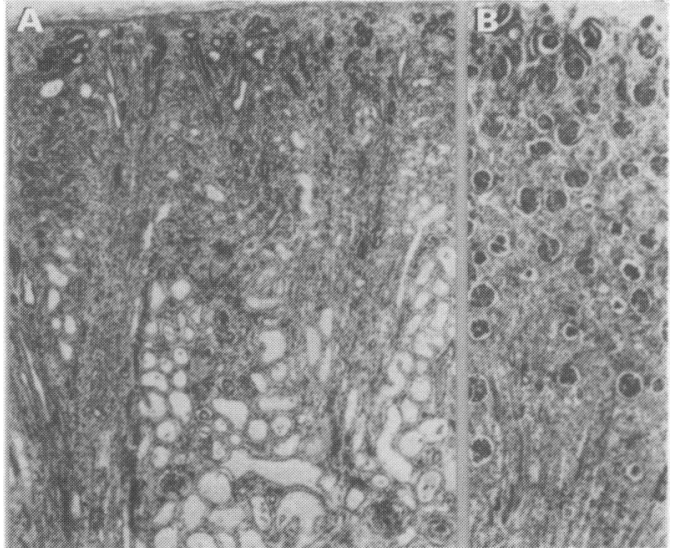

Figure 1 (A) The kidney from the case showing paucity of glomeruli and dilated tubules. (B) Normal kidney at 29 weeks'gestation (both stained with haematoxylin and $\operatorname{eosin} \times 30$ ).

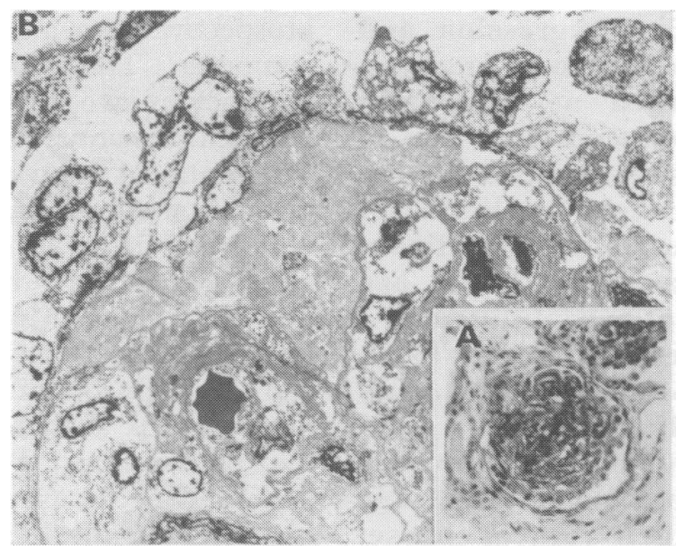

Figure 2 (A) Mesangial sclerosis in a glomerulus from the deep cortex (periodic acid Schiff 1 150). (B) Electron micrograph showing mesangial sclerosis, amorphous material in the subepithelial space, and detached epithelial cells (uranyl acetate lead citrate $\times 1800$ ).

deposition of filamentous material between the endothelium and the basement membrane proper, and there was collapse of capillary loops and sclerosis of the mesangium. In the smaller, more immature glomeruli there was a mass of amorphous material lodged between the epithelium and the basement membrane. The lamina densa was well developed.

\section{Discussion}

The interest of this case lies both in the unusual clinical presentation and the pathological findings. In our opinion the histological appearance indicates that the renal failure in this case was a disease process commencing in utero. At 29 weeks' gestation there are normally 9-10 layers of mature glomeruli and the nephrogenic blastema is prominent (fig $1 \mathrm{~B}$ ).$^{2}$ In our case (fig 1A) there were 4-5 layers of glomeruli, many of which were immature, which corresponds to the stage of development at about 20-22 weeks, and the blastema was thin and disordered. The appearance on electron microscopy (fig 2B) indicates that those glomeruli that were capable of function were suffering from hyperfiltration injury, with trapping of large molecules (including the IgM and C3 seen on immunostaining) in a leaking glomerulus. ${ }^{3}$ It appears that a pathological process, probably a specific glomerular disease, had curtailed the formation of new glomeruli during the last seven weeks in utero.

Although there are many possible causes of renal failure in preterm neonates, in this case the insidious onset and relentless progression are unusual features. Possible acquired causes in a preterm infant are antenatal hypoxia due to pre-eclampsia or abruptio placentae, intrapartum asphyxia, polycythaemia, emboli from a catheter, or gentamicin or pancuronium toxicity. However these would all cause a more acute onset of renal failure. More importantly, no disease acquired during or after birth could have had this profound effect on development.

Many congenital causes of renal insufficiency can be excluded on morphological grounds. These include malformations of the lower urinary tract, Finnish type congenital nephrotic syndrome, polycystic disease, and renal dysplasia. The lesion in the glomeruli bears some resemblance to that seen in congenital syphilis ${ }^{4}$ but this can be excluded on maternal serology, and by the absence of immune deposits.

The renal lesion most closely fits into the category of idiopathic diffuse mesangial sclerosis (DMS). ${ }^{5}$ This is a poorly characterised condition, probably of heterogenous aetiology, and can be familial. It is rare in infancy ${ }^{4}$ and is associated with a less rapidly fatal course than in our case. It has not been described in appreciably preterm infants. However the glomeruloscerotic lesions are similar to ours, and the infants with DMS who present early and survive for a few months do show an excess of immature glomeruli. ${ }^{467}$ In one 15 month infant there was also perpheral IgM and C3 staining in the glomeruli, without electron dense deposits on electron microscopy. 8 The precise pathogenetic mechanism remains obscure, in our case as in other cases of DMS.

We believe that this is an example of the influence on development of disease acquired during fetal life. From a practical point of view it emphasises the importance of reaching a pathological diagnosis in cases of neonatal renal failure.

We gratefully acknowledge the opinions given on the case by Professor R A Risdon of the Hospital for Sick Children, Great Ormond Street, London, and by Dr J Bernstein of the William Beaumont Hospital, Royal Oak, Michigan, USA.

1 Gruenwald P, Minh HN. Evaluation of body and organ weights in perinatal pathology. Am f Clin Pathol 1960; 34:247-53.

2 Dorovini-Zis K, Dolman CL. Gestational development of the brain. Arch Pathol Lab Med 1977;101:192-5.

3 Heptinstall RH. Pathology of the kidney. Vol 2. 3rd Ed. Boston: Little, Brown and Co, 1974.

4 McDonald R, Wigglinkhuizen J, Kaschula ROC. The nephrotic syndrome in very young infants. Am $\mathcal{f}$ Dis Child 1971,122:507-12

5 Habib R, Bois E. Heterogenety of early onset nephrotic syndromes in infants. Helvetica Paediatrica Acta 1973; 28:91-107.

6 Habib R, Gubler MC, Antignac C, Loirat C, Gangnadoux MF. Congenital or childhood nephrotic syndrome with diffuse mesangial sclerosis. Ann Pediatr (Paris) 1990; 37:73-77.

7 Sibley RK, Mahan J, Mauer SM, Vernier RL. A clinicopathological study of 48 infants with nephrotic syndrome. Kidney Int 1985;27:544-52.

8 Palomeque A, Calvo M, Esteva N, Darnell A. Nephrotic syndrome with diffuse mesangial sclerosis. An Esp Pediatr 1979;12 169-76. 